\title{
Immunohistochemical Diagnosis of Papillary Thyroid Carcinoma
}

Carol C. Cheung, M.D., Shereen Ezzat, M.D., Jeremy L. Freeman, M.D., Irving B. Rosen, M.D., Sylvia L. Asa, M.D., Ph.D.

Departments of Pathology and Laboratory Medicine (CCC, SLA), Medicine (Endocrinology; SE), Otolaryngology (JLF), and Surgery (IBR), The Freeman Center in Endocrine Oncology, Mount Sinai Hospital, University of Toronto, Toronto, Canada

In thyroid, the diagnosis of papillary carcinoma (PC) is based on nuclear features; however, identification of these features is inconsistent and controversial. Proposed markers of PC include HBME-1, specific cytokeratins (CK) such as $\mathrm{CK} 19$, and ret, the latter reflecting a ret/PTC rearrangement. We applied immunohistochemical stains to determine the diagnostic accuracy of these three markers. Formalin-fixed, paraffin-embedded tissue from 232 surgically resected thyroid nodules included 40 hyperplastic nodules $(\mathrm{NH}), 35$ follicular adenomas (FA), 138 papillary carcinomas (PC; 54 classical papillary tumors and 84 follicular variant papillary carcinomas [FVPC]), 4 follicular carcinomas (FC), 6 insular carcinomas (IC), 7 Hürthle cell carcinomas (HCC), and 2 anaplastic carcinomas (AC). HBME-1 and ret were negative in all $\mathrm{NH}$ and $\mathrm{FA}$; some of these exhibited focal CK19 reactivity in areas of degeneration. Half of the FC and AC exhibited HBME-1 staining but no positivity for CK19 or ret. In PC, $20 \%$ of cases stained for all three markers. Classical PC had the highest positivity with staining for HBME-1 in $70 \%$, CK19 in $80 \%$, and ret in $78 \%$. FVPC were positive for HBME-1 in 45\%, for CK19 in $57 \%$, and for ret in $63 \%$; only 7 FVPC were negative for all three markers. The six IC exhibited $67 \%$ staining for HBME-1 and $50 \%$ positivity for CK19 and ret. The seven HCC had $29 \%$ positivity for HBME-1 and CK19, and 57\% positivity for ret. This panel of three immunohistochemical markers provides a useful means of diagnosing PC. Focal CK19 staining may be found in benign lesions, but diffuse positivity is characteristic of PC. HBME-1 positivity indicates malignancy but not papillary differentia-

Copyright () 2001 by The United States and Canadian Academy of Pathology, Inc.

VOL. 14, NO. 4, P. 338, 2001 Printed in the U.S.A.

Date of acceptance: September 13, 2000.

Address reprint requests to: Sylvia L. Asa, M.D., Ph.D., Department of Pathology, University Health Network, 610 University Avenue, Toronto, Ontario M5G 2M9 Canada; e-mail: sylvia.asa@uhn.on.ca; fax: 416-946-6579. tion. Only rarely are all three markers negative in PC; this panel therefore provides an objective and reproducible tool for the analysis of difficult thyroid nodules.

KEY WORDS: Immunohistochemistry, Papillary thyroid carcinoma-cytology.

Mod Pathol 2001;14(4):338-342

Papillary carcinoma (PC) is the most common malignancy of thyroid follicular epithelium. Despite the propensity for lymphatic dissemination to cervical nodes, the majority of patients with these tumors, if appropriately treated, have an excellent long-term prognosis (1). Appropriate treatment then, rests primarily on the ability of the pathologist to render an accurate diagnosis. Historically, the identification of PC relied on the presence of papillary architecture. The current accepted diagnosis of this entity is based on nuclear features that include optical clearing, elongation, overlapping, micronucleoli, and irregular contours with grooves and pseudoinclusions (2-4). However, identification of these features remains, at times, controversial, and the distinction of papillary carcinoma from follicular adenoma can be difficult.

Proposed markers of thyroid malignancies include HBME-1, specific cytokeratins (CK) such as CK19, and ret; the latter two markers each identify a subpopulation of PC.

HBME-1 is a marker of mesothelial cells. Several studies have demonstrated its utility as a marker of malignant thyroid tumors of follicular epithelial derivation (5-7). HBME-1 positivity in thyroid follicular epithelial tumors is, therefore, indicative of malignancy, but it does not necessarily indicate papillary differentiation.

Papillary carcinomas have been shown to express CK19 with strong diffuse cytoplasmic reactivity (812). This marker is expressed focally in reactive thyroid follicular epithelium; however, most au- 
thors have not identified diffuse CK19 positivity in follicular adenomas or carcinomas $(8-10,12)$.

The ret/PTC family of chimeric oncogenes result from several rearrangements involving the ret gene of chromosome 10q (13-15); they are specific to papillary carcinomas and are present in up to $77 \%$ of these tumors (16). The protein product of the ret/PTC oncogenes all contain the intracellular tyrosine kinase domain of the normal ret protooncogene product and can therefore be immunohistochemically detected with an antibody to the carboxy terminus of ret $(16,17)$.

We applied this triad of immunohistochemical stains to a spectrum of thyroid lesions to determine their diagnostic accuracy.

\section{MATERIALS AND METHODS}

Formalin-fixed, paraffin-embedded tissue from 232 surgically resected thyroid specimens in the pathology files of Mount Sinai Hospital were classified histologically according to the criteria of LiVolsi (1). They included 40 cases of nodular hyperplasia $(\mathrm{NH})$. There were 35 follicular adenomas (FA) that were all noninvasive tumors with micro- and macrofollicular or solid architecture and tumors with oncocytic cytoplasm but no hyalinizing trabecular lesions or tumors with cytologic atypia. We examined 138 papillary carcinomas (PC), 54 with classical papillary architecture and 84 follicular variant papillary carcinomas (FVPC) with unequivocal nuclear features of papillary carcinoma. The four follicular carcinomas (FC) exhibited obvious capsular and/or vascular invasion and no nuclear features of papillary carcinoma. In addition, there were six insular carcinomas (IC) that had classical nesting architecture and widespread invasion; seven Hürthle cell carcinomas (HCC), also with extensive invasion; and two anaplastic carcinomas (AC).

All specimens were fixed in $10 \%$ neutral buffered formalin and embedded in serial 3-4 $\mathrm{mm}$ paraffin blocks; $4-\mu \mathrm{m}$-thick sections were stained with hematoxylin and eosin for histologic examination (18). Representative sections were then immunostained to localize HBME-1, CK19, and ret.

Immunostaining for HBME-1 was performed using a mouse anti-human monoclonal antibody (clone HBME-1; DAKO Corporation, Carpinteria, CA); negative controls replaced primary antibody with buffer. Four-micrometer-thick tissue sections were pretreated with $44 \%$ formic acid for 15 minutes at room temperature. After blocking endogenous peroxidase and nonspecific binding, the primary antibody at a dilution of 1:100 was incubated at room temperature for 60 minutes, followed by detection with the ultrastreptavidin system (Signet, Dedham, MA).
Immunostaining for CK19 was performed using a mouse anti-human monoclonal antibody (clone b170; Novocastra, Newcastle upon Tyne, UK); negative controls replaced primary antibody with buffer. Four-micrometer-thick tissue sections were pretreated with pepsin for 10 minutes at room temperature. After blocking endogenous peroxidase and nonspecific binding, the primary antibody at a dilution of 1:150 was incubated at room temperature for 60 minutes, followed by detection with the ultrastreptavidin system (Signet).

Immunostaining for ret was performed using a rabbit polyclonal IgG antibody to the carboxy terminus of ret (Santa Cruz Biotechnology, Inc, Santa Cruz, CA); negative controls replaced primary antiserum with nonimmune rabbit serum. Fourmicrometer-thick tissue sections were pretreated with $44 \%$ formic acid for 15 minutes at room temperature. After blocking endogenous peroxidase and nonspecific binding, the primary antibody at a dilution of 1:200 incubated at room temperature for 60 minutes, followed by detection with the ultrastreptavidin system (Signet).

\section{RESULTS}

HBME-1 decorated a significant proportion of malignancies, including papillary carcinomas, both classical and follicular variants (Fig. 1, a-b), and follicular carcinoma (Fig. 1c). In some cases of PTC, the staining was predominantly luminal (Fig. 1a). CK19 displayed several patterns of immunoreactivity. In some lesions, there was focal strong cytoplasmic positivity; this was associated predominantly with degeneration. In papillary carcinomas, there was diffuse moderate to strong cytoplasmic reactivity (Fig. 2a), which was also seen in FVPC (Fig. 2b). Follicular lesions, including nodular hyperplasias (Fig. 2c), adenomas, and carcinomas were negative except around areas of degeneration. Ret staining was generally weak and focal. This predominantly cytoplasmic stain was identified only in papillary carcinomas, including classical variants (Fig. 3a), follicular variants (Fig. 3b), and solid variants (Fig. 3c).

The results of immunostains are tabulated by diagnosis in Table 1. All benign thyroid follicular nodules, including hyperplastic lesions in sporadic nodular goiter (nodular hyperplasia, $\mathrm{NH}$ ) and all 35 follicular adenomas, were negative for HBME-1 and ret. Some of the benign follicular lesions stained for CK19, but the pattern was consistent with focal CK19 reactivity in areas of degeneration, usually at the site of previous fine-needle aspiration biopsy. Two of four follicular carcinomas and one of two anaplastic carcinomas exhibited HBME-1 staining but had no positivity for CK19 or ret. 
Papillary carcinomas were frequently positive for these markers. All three stains were positive in $20 \%$ of papillary carcinomas. Classical PC that were characterized by papillary architecture had the highest positivity, with 70\% HBME-1, 80\% CK19, and $78 \%$ ret staining. Follicular-variant PCs were positive for HBME-1 in 45\%, for CK19 in 57\%, and for ret in $63 \%$; only 7 of 84 tumors classified as PC on the basis of cytologic atypia were negative for all three markers.

The six insular carcinomas exhibited $67 \%$ staining for HBME-1 and 50\% positivity for CK19 and ret. Among seven malignancies composed of Hürthle cells, 2 (29\%) were positive for HBME-1; four (57\%) were positive for ret as described elsewhere (17), indicating papillary differentiation, and of these, two were also positive for CK19. Two lesions classified as Hürthle cell carcinoma were negative for all three markers.

\section{DISCUSSION}

HBME-1 is a monoclonal antibody directed against an unknown epitope on mesothelial cells. Several studies have shown its utility as a marker in the identification of malignant follicular epithelial lesions of the thyroid (5-7). Its staining pattern is predominantly membranous with variable cytoplasmic positivity. Our study demonstrated HBME-1 immunoreactivity in $54 \%$ of malignant lesions of follicular epithelial derivation. All benign lesions were negative. HBME-1 positivity was present in every type of follicular epithelial malignancy; this included 55\% of PC. Our data indicate that HBME-1 reactivity in a suspected adenoma or a dominant hyperplastic nodule should provoke a search for other features of malignancy, including capsular or vascular invasion; further cytologic and immunohistochemical evaluation will help deter-
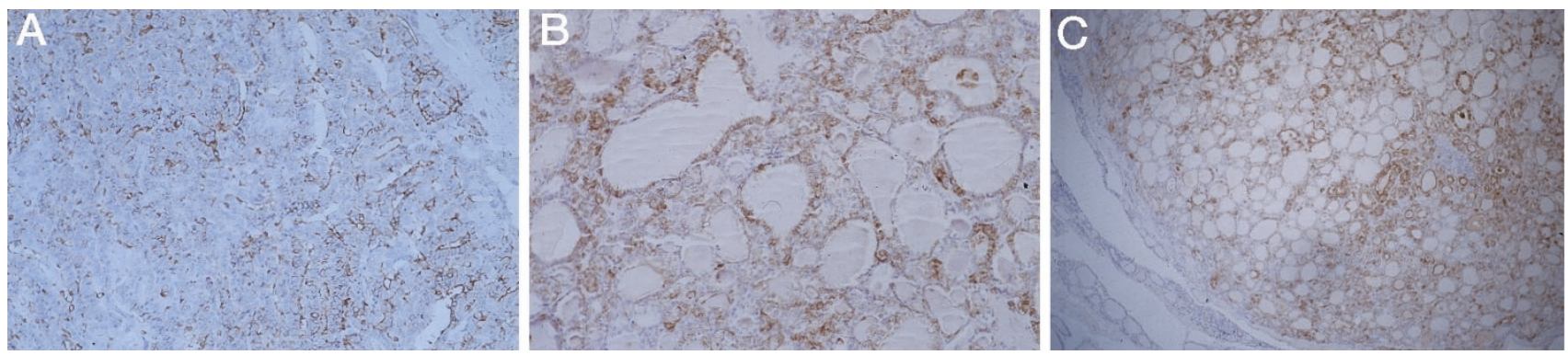

FIGURE 1. Immunohistochemistry for HBME-1. A, Classic papillary carcinoma with beautiful complex papillae exhibits a membrane staining pattern. B, Follicular variant papillary carcinoma shows strong staining with focal luminal accentuation. C, Follicular carcinoma exhibits HBME-1 immunoreactivity.
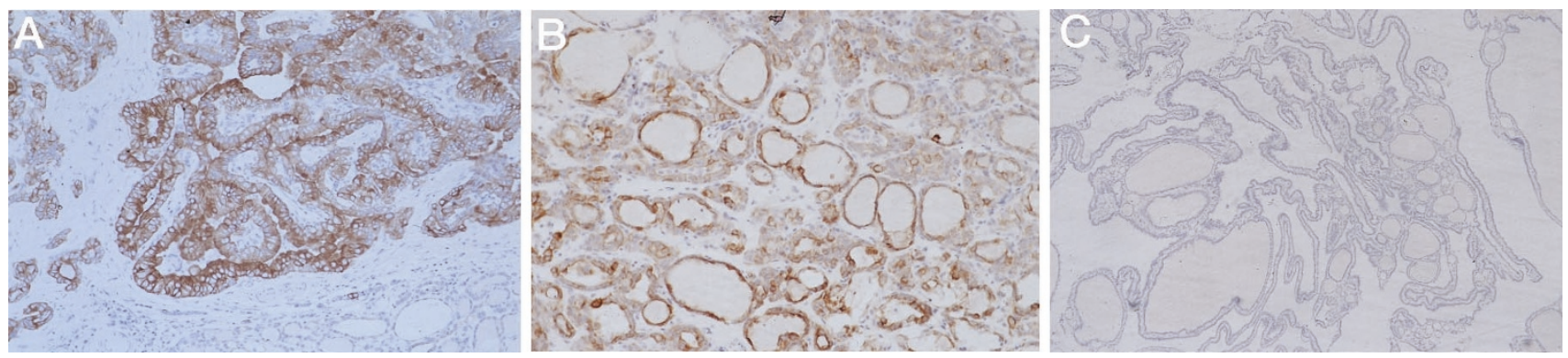

FIGURE 2. Immunohistochemistry for CK19. A, Diffuse immunoreactivity is strong in a classical papillary carcinoma. B, Follicular variant papillary carcinoma also exhibits diffuse cytoplasmic immunoreactivity. C, A hyperplastic lesion is entirely negative for CK19.
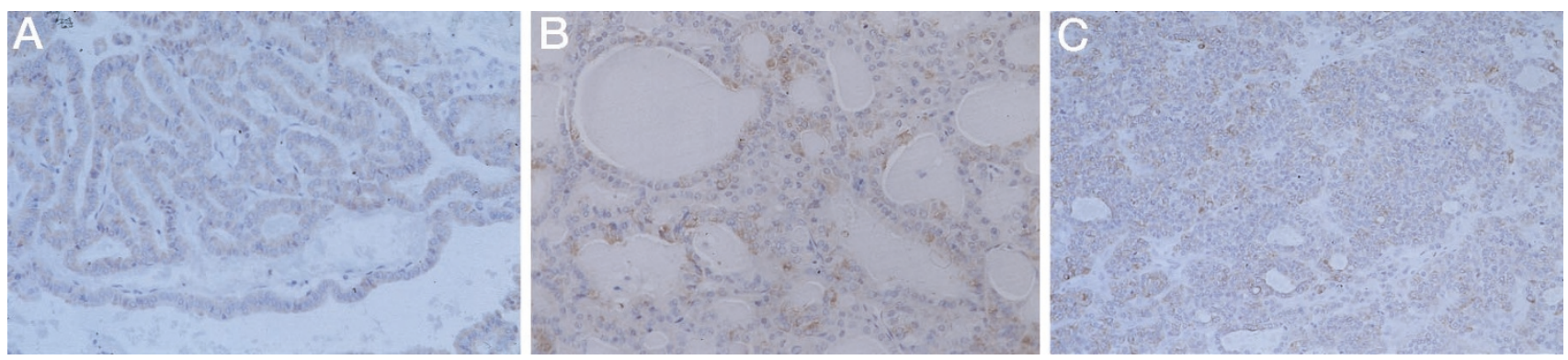

FIGURE 3. Immunohistochemistry for ret. A, Classical papillary carcinoma exhibits cytoplasmic positivity for ret, indicating the expression of a ret/ PTC gene rearrangement. B, Follicular variant papillary carcinoma is also positive for ret. C, A solid-variant papillary carcinoma exhibits focal reactivity for ret. 


\begin{tabular}{|c|c|c|c|c|}
\hline \multirow[b]{2}{*}{ Diagnosis } & \multicolumn{3}{|c|}{ Marker } & \multirow{2}{*}{$\begin{array}{c}\text { All Three } \\
\text { Negative (\%) }\end{array}$} \\
\hline & $\begin{array}{c}\text { HBME- } 1 \\
(\%)\end{array}$ & CK19 (\%) & $\begin{array}{l}\text { Ret } \\
(\%)\end{array}$ & \\
\hline Nodular hyperplasia $(n=40)$ & 0 & 8 focal (20) & 0 & $32(80)$ \\
\hline Follicular adenoma $(n=35)$ & 0 & $\begin{array}{l}1 \text { diffuse (3) } \\
6 \text { focal (17) }\end{array}$ & 0 & $28(80)$ \\
\hline Papillary carcinoma $(n=138)$ & $76(55)$ & $91(66)$ & $95(69)$ & $9(6.5)$ \\
\hline Classical variant $(n=54)$ & $38(70)$ & $43(80)$ & $42(78)$ & $2(4)$ \\
\hline Follicular variant $(n=84)$ & $38(45)$ & $48(57)$ & $53(63)$ & $7(8)$ \\
\hline Follicular carcinoma $(n=4)$ & $2(50)$ & 0 & 0 & $2(50)$ \\
\hline Insular carcinoma $(n=6)$ & $4(67)$ & $3(50)$ & $3(50)$ & 0 \\
\hline Hürthle cell carcinoma ( $n=7$ ) & $2(29)$ & $2(29)$ & $4(57)$ & $2(29)$ \\
\hline $\begin{array}{l}\text { Anaplastic carcinoma }(n=2) \\
\text { Total }(n=232)\end{array}$ & $1(50)$ & 0 & 0 & $1(50)$ \\
\hline
\end{tabular}

mine whether or not the lesion is a papillary carcinoma.

The ret gene, located on Chromosome 10q, encodes a transmembrane tyrosine kinase receptor (13-15). Ret is normally expressed in neural crestderived cells; normal thyroid follicular epithelial cells do not express ret (19). However, in the majority of PCs, a gene rearrangement occurs that places the intracellular domain of the ret gene under the transcriptional control of one of several genes that are expressed in thyroid follicular epithelium (16). This chimeric gene, the ret/PTC oncogene, is specific to papillary carcinomas and encodes a protein product that contains the cytoplasmic portion of ret (13-15). Therefore, immunohistochemical detection of the carboxy terminus of the ret protein in thyroid follicular epithelial lesions serves as a reliable marker for papillary carcinoma (16). In our study, ret immunoreactivity was demonstrated in $69 \%$ of papillary carcinomas. All benign nodules were nonimmunoreactive for ret. Although ret has been occasionally reported in nonneoplastic follicular cells in chronic lymphocytic thyroiditis $(16,20)$ and we have seen immunoreactivity in this setting (unpublished data), this is not usually a dilemma in the histological diagnosis of malignancy because this is a diffuse process rather than a discrete nodule.

Insular carcinomas are poorly differentiated carcinomas of follicular epithelial origin and are so named because of their distinctive architecture. Many authors believe that insular carcinomas dedifferentiate from well-differentiated carcinomas (1, 2). In fact, careful histologic examination supports this, as most cases of insular carcinoma will contain a well-differentiated component. In our study, the insular carcinomas that were immunoreactive for ret all histologically exhibited focal areas consistent with papillary carcinoma; these tumors most likely dedifferentiated from ret-expressing papillary carcinomas.

Our study included 7 Hürthle cell carcinomas. These were tumors that on hematoxylin and eosin examination were infiltrative lesions without evidence of a well-defined capsule. Two cases were immunoreactive for HBME-1, a finding that supports their malignant nature. Recently, the molecular basis of Hürthle cell papillary carcinomas was elucidated using the detection of ret/PTC expression by RT-PCR in a series of 50 Hürthle cell tumors (17). It seems reasonable then, that the four "Hürthle cell carcinomas" in our series exhibiting ret immunoreactivity are in fact Hürthle cell papillary carcinomas.

A number of previous studies have examined the immunoreactivity profiles of various cytokeratins subtypes (8-12). CK19 has been shown to be a sensitive marker for papillary carcinomas, usually resulting in diffuse cytoplasmic positivity. Unfortunately, CK19 is also expressed focally in normal thyroid epithelium, Hashimoto's thyroiditis, and some benign tumors. Focal strong staining was present in 14 of 75 benign lesions; the majority of the immunoreactivity occurred in areas of degeneration, indicating the reactive nature of CK19 positivity. Nevertheless, diffuse positivity may have diagnostic value. In our study, diffuse CK19 immunoreactivity was demonstrated in $66 \%$ of PCs. Diffuse positivity was also present in other follicular epithelial malignancies, including three insular carcinomas and two Hürthle cell carcinomas, however, these also exhibited staining for ret, indicating papillary differentiation. One follicular adenoma exhibited diffuse positivity for CK19, and the significance of this remains unclear; this tumor was not invasive and did not exhibit the nuclear atypia of papillary carcinoma; however, these features are not always predictive of benign behavior, and long-term follow-up may prove the value of this finding.

In summary, our data indicate that immunoreactivity for HBME-1 represents a reliable indicator of malignancy in nodules of follicular epithelial derivation. However, this marker only decorates a subset of thyroid malignancies, so that the malignant potential of a lesion that is negative for HBME-1 cannot be established. These conclusions are in 
agreement with those of Sack et al. (6). Ret immunoreactivity is a reliable diagnostic marker of papillary thyroid carcinoma, but again, lack of staining does not exclude the diagnosis because only $69 \%$ of tumors in this series stained for this marker. Staining for CK19 requires qualitative evaluation. Diffuse positivity represents a good, supportive immunohistochemical marker of papillary carcinomas; however, focal staining is often found in other lesions, including benign nodules, in areas of degeneration. Positivity for all three markers is very specific for papillary carcinoma.

Along with careful histologic evaluation, this panel of three immunohistochemical markers provides a useful and objective means of diagnosing PC. HBME-1 positivity indicates a malignancy, whereas diffuse CK-19 and/or ret positivity confirm papillary differentiation. Although many PCs are not positive for all three stains, only rarely are all three markers negative; this panel therefore provides a tool for the analysis of difficult thyroid nodules.

\section{REFERENCES}

1. LiVolsi VA. Surgical pathology of the thyroid. Vol. 5. Philadelphia: WB Saunders; 1990.

2. Rosai J, Carcangiu ML, DeLellis RA. Tumors of the thyroid gland. Atlas of tumor pathology. 3rd series. Fascicle 5. Washington, DC: Armed Forces Institute of Pathology; 1992.

3. Hapke MR, Dehner LP. The optically clear nucleus. A reliable sign of papillary carcinoma of the thyroid? Am J Surg Pathol 1979;3:31-8.

4. Hedinger C, Williams ED, Sobin LH. Histological typing of thyroid tumours. World Health Organization International Histological Classification of Tumours. 2nd ed. Berlin: Springer-Verlag; 1988.

5. Miettinen M, Karkkainen P. Differential reactivity of HBME- 1 and CD15 antibodies in benign and malignant thyroid tumours. Preferential reactivity with malignant tumours. Virchows Arch 1996;429:213-9.

6. Sack MJ, Astengo-Osuna C, Lin BT, Battifora H, Li Volsi VA. HBME-1 immunostaining in thyroid fine-needle aspirations: a useful marker in the diagnosis of carcinoma. Mod Pathol 1997;10:668-74.
7. Greenspan FS. The role of fine-needle aspiration biopsy in the management of palpable thyroid nodules. Am J Clin Pathol 2000;108(1 Suppl):26-30.

8. Fonseca E, Nesland J, Hoie J, Sobrinho-Simoes M. Pattern of expression of intermediate cytokeratin filaments in the thyroid gland: an immunohistochemical study of simple and stratified epithelial-type cytokeratins. Virchows Arch 1997; 430:239-45.

9. Raphael SJ, Apel RL, Asa SL. Brief report: detection of highmolecular-weight cytokeratins in neoplastic and nonneoplastic thyroid tumors using microwave antigen retrieval. Mod Pathol 1995;8:870-2.

10. Uchida G, Nakayama I, Noguchi S. An immunohistochemical study of cytokeratin and vimentin in benign and malignant thyroid lesions. Acta Pathol Jpn 1989;39:169-75.

11. Miettinen M, Kovatich AJ, Kärkkäinen P. Keratin subsets in papillary and follicular thyroid lesions. A paraffin section analysis with diagnostic implications. Virchows Arch 1997; 431:407-13.

12. Baloch ZW, Abraham S, Roberts S, Li Volsi VA. Differential expression of cytokeratins in follicular variant of papillary carcinoma: an immunohistochemical study and its diagnostic utility. Hum Pathol 1999;30:1166-71.

13. Fusco A, Grieco M, Santoro M, Berlingieri MT, Pilotti S, Pierotti MA, et al. A new oncogene in human thyroid papillary carcinomas and their lymph-nodal metastases. Nature 1987;328:170-2.

14. Jhiang SM, Mazzaferri EL. The ret/PTC oncogene in papillary thyroid carcinoma [review]. J Lab Clin Med 1994;123:331-7.

15. Santoro M, Carlomagno F, Hay ID, Herrmann MA, Grieco M, Melillo R, et al. Ret oncogene activation in human thyroid neoplasms is restricted to the papillary cancer subtype. J Clin Invest 1992;89:1517-22.

16. Sugg SL, Ezzat S, Rosen IB, Freeman J, Asa SL. Distinct multiple ret/PTC gene rearrangements in multifocal papillary thyroid neoplasia. J Clin Endocrinol Metab 1998;83: 4116-22.

17. Cheung CC, Ezzat S, Ramyar L, Freeman J, Asa SL. Molecular basis of Hurthle cell papillary thyroid carcinoma. J Clin Endocrinol Metab 2000;85:878-82.

18. Fink A, Tomlinson G, Freeman JL, Rosen IB, Asa SL. Occult micropapillary carcinoma associated with benign follicular thyroid disease and unrelated thyroid neoplasms. Mod Pathol 1996;9:816-20.

19. Nakamura T, Ishizaka $Y$, Nagao M, Hara M, Ishikawa T. Expression of the ret proto-oncogene product in human normal and neoplastic tissues of neural crest origin. J Pathol 1994;172:255-60.

20. Wirtschafter A, Schmidt R, Rosen D, Kundu N, Santoro M, Fusco A, et al. Expression of the RET/PTC fusion gene as a marker for papillary carcinoma in Hashimoto's thyroiditis. Laryngoscope 1997;107:95-100. 